\title{
The Use of CFSE-like Dyes for Measuring Lymphocyte Proliferation: Experimental Considerations and Biological Variables
}

\author{
B.J.C. Quah ${ }^{1}$, A.B. Lyons ${ }^{2}$, C.R. Parish ${ }^{1}$ * \\ ${ }^{1}$ Department of Immunology, John Curtin School of Medical Research, Australian National University, \\ Canberra, ACT, 2601, Australia \\ ${ }^{2}$ School of Medicine, University of Tasmania, Hobart, Tasmania, Australia
}

\begin{abstract}
The measurement of CFSE dilution by flow cytometry is a powerful experimental tool to measure lymphocyte proliferation. CFSE fluorescence precisely halves after each cell division in a highly predictable manner and is thus highly amenable to mathematical modelling. However, there are several biological and experimental conditions that can affect the quality of the proliferation data generated, which may be important to consider when modelling dye dilution data sets. Here we overview several of these variables including the type of fluorescent dye used to monitor cell division, dye labelling methodology, lymphocyte subset differences, in vitro versus in vivo experimental assays, cell autofluorescence, and dye transfer between cells.
\end{abstract}

Keywords and phrases: Cell proliferation, CFSE, Flow cytometry

Mathematics Subject Classification: 92-02, 92C37, 92C99, 97M60

\section{Introduction}

CFSE dilution is one of the most commonly used measures of lymphocyte proliferation in immunological studies. The use of CFSE in measuring lymphocyte proliferation began with our studies using the fluorescent dye in lymphocyte migration analyses in mouse models [1]. CFSE was ideally suited for this application, labelling cells with a very bright stable fluorescence that had very minimal fluorescence variation throughout the labelled cell population and had low cell toxicity. After adoptive transfer into mice CFSE-labelled lymphocytes could therefore be identified quite discretely from host cells using flow cytometry over long periods of time. Bruce Lyons, repeating similar migration experiments in our laboratory, fortuitously noted that CFSE-labelled B cells transferred into very young mice appeared to undergo serial halving of dye fluorescence intensity, indicative of cell proliferation [2,3]. Indeed, Lyons and Parish went on to confirm, using Hoechst fluorescence quenching by BrDU, that daughter cells in a dividing CFSE-labelled population of lymphocytes had received half the amount of CFSE from their parent cells [2].

In hindsight, the same key attributes of CFSE that make it an ideal cell tracking dye in in vivo migration assays also make it ideal for proliferation analysis. The high fluorescence that can be achieved

${ }^{*}$ Corresponding author. E-mail: Christopher.Parish@anu.edu.au 


\section{Data components}

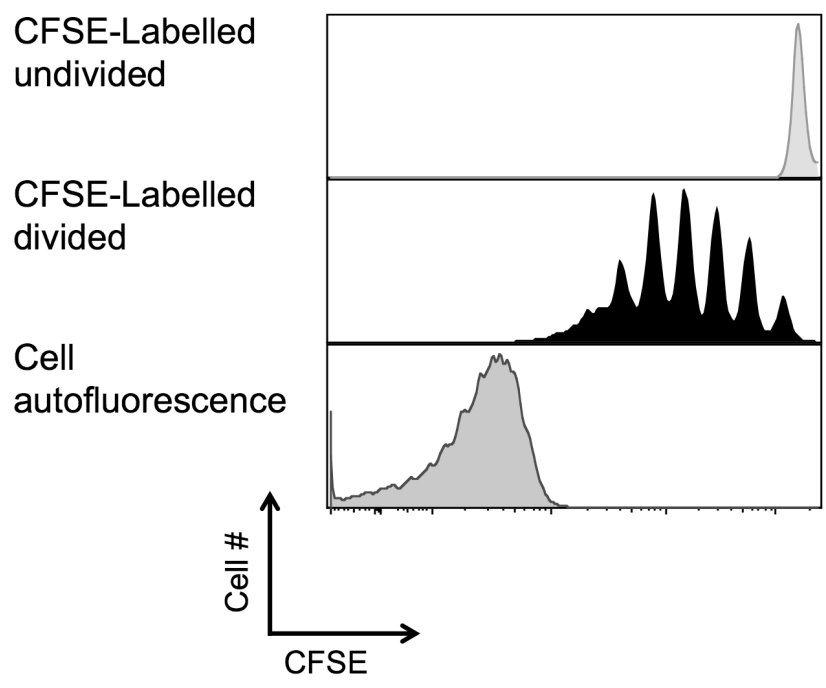

Combined data

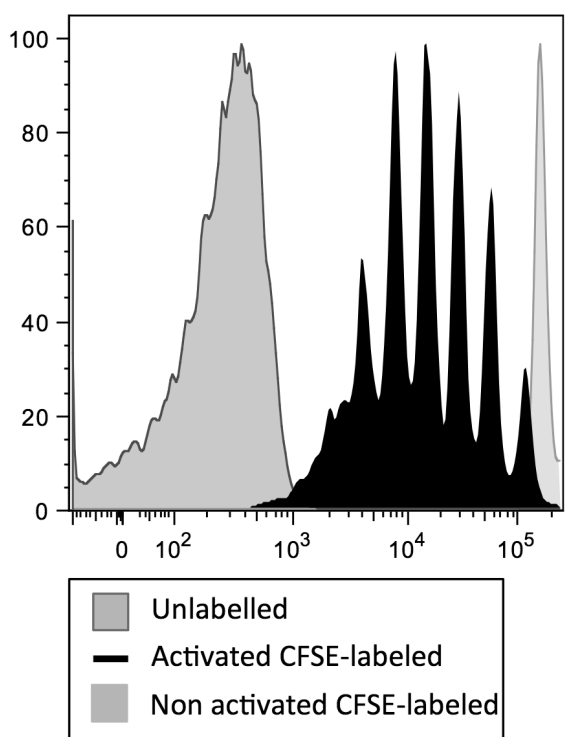

Figure 1. CFSE-dilution as a measure of lymphocyte proliferation. Spleen cells from mice were labelled with $40 \mu \mathrm{M}$ of CFSE in RPMI1640 medium supplemented with $10 \%$ FCS and cells stimulated with multiple mitogens [7] in in vitro culture for 4 days and T cells (CD90.2 ${ }^{+}$) analysed by flow cytometry for CFSE dilution. In addition, unstimulated CFSE-labelled T cells and unlabelled T cells were also cultured for 4 days to monitor undivided cell fluorescence and cell autofluorescence.

with CFSE-labelled cells means that it takes several rounds of division before the dye dilutes to the equivalence of cell autofluorescence, allowing several divisions to be detected. The minimal variation in dye labelling of lymphocytes (lymphocytes labelled with CFSE can have a fluorescence standard deviation of $25 \%$ or less than the mean fluorescence intensity across a broad range of fluorescence levels) coupled with high and uniform stability of labelling across a broad range of fluorescence intensities results in each cell division being associated with a discrete shift in the level of fluorescence. In addition, to maximise fluorescent peak resolution it is possible to pre-sort cells to have a very narrow CV of CFSE fluorescence before initiation of division [4]. Although there have been reports that CFSE labelling can be toxic for cells $[5,6]$, when cells are labelled in appropriate buffers cell toxicity is very low $[5,7]$. A typical division histogram of mouse T cells, using current CFSE cell-labelling methodologies [7-9], is depicted in Figure 1. This figure highlights the highly uniform fluorescent labelling that results in discrete divisions being achievable with CFSE and also shows the importance of including non-activated CFSE-labelled cells and unlabelled cells to monitor undivided cell fluorescence and cell autofluorescence which indicate the extent of dye dilution/division number that is possible for any given assay. It should be noted, however, that in the example shown in Figure 1 the fluorescence of the non-activated CFSE-labelled cells is slightly higher than the fluorescence of the non-dividing cells in the proliferating population. This difference varies from experiment to experiment for reasons that are unclear, although the data shown in Figure 1 represents an extreme example of this phenomenon. Nevertheless, it is an important variable that should be considered when mathematically modelling CFSE proliferation data. 
The application of CFSE to cell division studies has now been used for a number of species other than mouse and human, such as teleosts [10]. In addition, cells other than lymphocytes have been shown to be amenable to CFSE analysis, such as hemopoietic stem cells [4], myeloid leukemic progenitors [11] and bacteria [12]. A major application has been the analysis of cell differentiation, which has shown that for both $\mathrm{B}$ and $\mathrm{T}$ lymphocytes, there is a clear link between cell cycle number and acquisition of differentiation markers, such as cell surface molecules and secreted cytokines [13-15]. Other studies have also shown that CFSE analysis can be performed using cell nuclei prepared from dividing lymphocytes, allowing analysis of the nuclear transcription factors that control division and differentiation [16]. In addition to lymphocyte behaviour in immune processes, CFSE analysis has also been used to examine the modification of lymphocyte division by drugs targeting cell kinases [17-19].

Since CFSE fluorescence precisely halves after each cell division in a highly predictable manner [7] data obtained from CFSE proliferation assays is highly amenable to mathematical modelling [19-27]. From a biological point of view, mathematical models offer a way to examine how the immune response develops in a temporal and quantitative way. It may be useful in predicting the success of an immune response based on key correlates of lymphocyte development such as the extent of lymphocyte subset proliferation. Furthermore, the use of modeling to predict distinct CFSE-based division peaks from experimental data that has poorly resolved division profiles can help to correlate phenotypes more precisely with the division history of cells.

There are several biological and experimental conditions we have noted over the years that can affect the quality of the proliferation data generated and, consequently, may be important to consider when modelling dye dilution data sets. These biological and experimental variables became more apparent when additional, CFSE-like, fluorescent dyes became available to monitor lymphocyte proliferation. Here we overview several of these variables including the type of fluorescent dye used to monitor cell division, dye labelling methodology, lymphocyte subset differences, in vitro versus in vivo experimental assays, cell autofluorescence, and dye transfer between cells.

\section{Fluorescent dyes used to measure lymphocyte proliferation}

The reason CFSE has such outstanding cell labelling properties is essentially due to two key chemical attributes of the dye $[5,7]$. The first is the two acetate groups that allow the dye to readily cross the plasma membrane of cells, but are subsequently enzymatically removed by intracellular esterases. This cleavage has two effects: the dye becomes much less membrane permeant and thus accumulates rapidly within the cell and the dye becomes highly fluorescent. The net result is a rapid accumulation of fluorescent CFSE molecules within viable cells giving a high level of cell fluorescence. The second key chemical attribute of the dye is the succinimidyl group of CFSE that is responsible for the retention of the fluorescent dye within cells, the amine reactive succinimidyl group covalently attaching the dye to cellular proteins. Since many proteins within the cell are long lived, the covalent attachment of the dye results in stably fluorescent cells, with high turnover proteins that are CFSE labelled being eliminated by catabolism within the first 24 hours after labelling.

While many fluorescent dyes have been used as an alternative to CFSE in experimental assays, the most successful of these alternate dyes are those that have similar chemical properties to CFSE. These include SNARF-1 (Life Technologies), also an esterase activated succinimidyl containing dye, with relatively broad emission properties ( $550 \mathrm{~nm}-750 \mathrm{~nm}$; i.e., across the PE and PerCP channels) and excited by a $488 \mathrm{~nm}$ laser; DDAO-SE (Life Technologies), a succinimidyl containing dye that has an emission peak at $\sim 657 \mathrm{~nm}$ (equivalent to APC) and is excited by a $633 \mathrm{~nm}$ laser; Cell Proliferation Dye eFluor 670 (CPD, ebioscience), an amine reactive dye that like DDAO-SE has an emission peak equivalent to APC and is excited by a $633 \mathrm{~nm}$ laser; and CellTrace Violet (CTV, Life Technologies), which is also an esterase activated succinimidyl containing dye, that has an emission peak at $\sim 455 \mathrm{~nm}$ (equivalent to Pacific Blue) and is excited by a $405 \mathrm{~nm}$ laser.

In addition, there are several other dyes chemically quite distinct from CFSE that have been used with reasonable success for cell proliferation analyses. A notable example is the lipophilic dyes, such as 
CFSE labeled cells

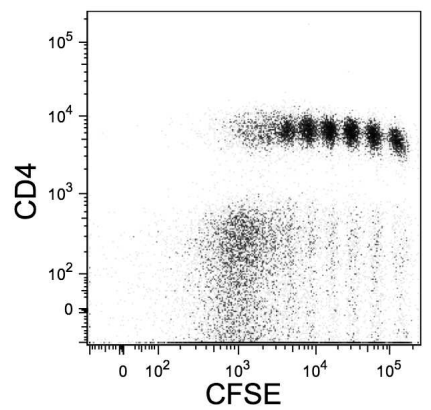

CTV labeled cells
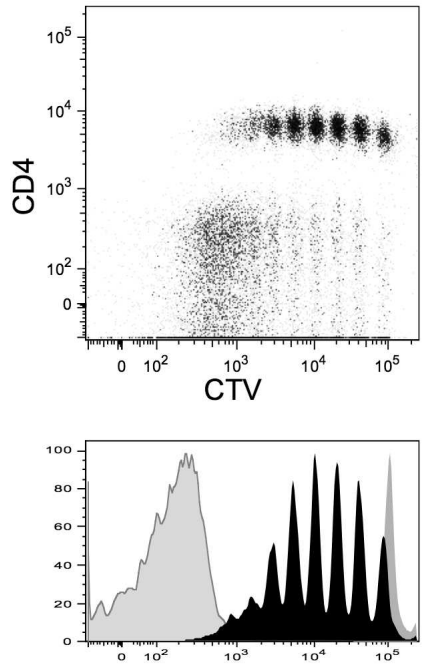

CD4+ T cells
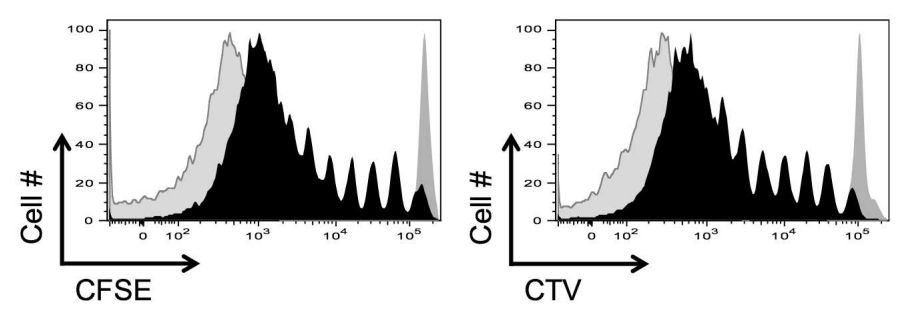

$\mathrm{CD}^{+}$ T cells

Figure 2. Comparison of CFSE-dilution and CTV-dilution in measuring lymphocyte proliferation. Spleen cells from mice were labelled with $40 \mu \mathrm{M}$ CFSE or CTV in RPMI1640 medium supplemented with 10\% FCS and cells stimulated with multiple mitogens [7] in in vitro culture for 4 days and then CD4+ and CD8+ T cells (CD90.2 ${ }^{+}$) analysed by flow cytometry for dye dilution. In addition, unstimulated CFSE-labelled spleen cells and unlabelled spleen cells were also cultured for 4 days to monitor undivided cell fluorescence and cell autofluorescence.

PKH-26 (Sigma-Aldrich) or the equivalent dye DiI (Life Technologies), which are rapidly incorporated into the lipid bilayer of membranes forming strong non-covalent interactions with surrounding lipid tails. PKH-26/DiI has an emission peak at $~ 567 \mathrm{~nm}$ (equivalent to PE) and is excited by a $488 \mathrm{~nm}$ laser.

While many of these dyes have provided a useful alternate to CFSE, since they emit at wavelengths compatible with fluorochromes emitting in the CFSE/FITC emission range, most are inferior to CFSE in detecting cell division. Invariably this is due, among other differences, to these dyes being unable to resolve discrete division peaks as well as CFSE [7,28-31]. In our experience so far, only one dye, Cell Trace Violet (CTV), is comparable to CFSE in measuring lymphocyte division [7]. Indeed, when we have performed a comprehensive comparison of lymphocytes labelled with CFSE and CTV [7], CTVlabelling performs on a par with CFSE-labelling in almost every aspect of cell proliferation. This is highlighted in Figure 2 which shows the clarity of the distinct division peaks detected by CFSE and CTV in proliferating $\mathrm{T}$ cell populations. Therefore, we would recommend CTV as the dye of choice for lymphocyte proliferation studies in which, due to spectral overlap, other flow cytometry assays are not compatible with CFSE fluorescence. 


\section{Lymphocyte dye labelling methods and analytical consideration}

The methodology used to label lymphocytes with proliferation-measuring dyes can have a severe impact on the fluorescence variability and viability of labelled cells as well as the number of possible divisions detectable. In this section we overview several important technical considerations when labelling cells with dyes like CFSE and CTV.

\subsection{Dye preparation}

Since CFSE and CTV will react upon exposure to aqueous solutions it is essential that stock solutions are prepared in anhydrous DMSO. Aliquots (both powder and solution) that are stored below room temperature should be brought to room temperature before exposure to air to avoid water condensation occurring and reacting with the dye.

\subsection{Dye concentration}

The concentration of dye used to label lymphocytes will determine the fluorescence level of the labelled cells are and therefore how many divisions can be resolved. In the case of CFSE and CTV, there is a linear relationship between the concentration of the dye used and the fluorescence intensity of the labelled cells [32-36]. The dye concentration used is ultimately limited by the amount of spectral "spill over" that occurs into spectrally adjacent detectors of the flow cytometer (since this "spill over" increases with dye fluorescence intensity) and also the cellular toxicity that can be associated with higher dye concentrations $[5,6]$. Toxicity becomes less limiting when high levels of reactive amino groups are present in the labelling buffer ([7] see below). Our current labelling protocols involve exposing cells to dye concentrations ranging from 10 to $80 \mu \mathrm{M}$, these labelling conditions resulting in optimum proliferation peak resolution, with up to 10 to 12 divisions being detectable at higher dye loading concentrations [7].

\subsection{Technique of mixing cells with dye}

Since CFSE and CTV rapidly accumulate within cells upon dye exposure, it is critical that the dye is mixed throughout the cell suspension as quickly as possible to ensure homogeneous cell labelling. Indeed, to achieve low variance of fluorescent peaks in cell proliferation assays initial uniform labelling of cell populations is essential. There are several techniques described to accomplish this [5,9], with uniform labelling being quite easily achieved by vortexing the cells whilst adding the concentrated dye to a 1-2 ml suspension of cells.

\subsection{Length of cell-dye incubation}

Labelling of lymphocytes with CFSE and CTV essentially reaches saturation levels after 5 min incubation of the dyes with cells at $20^{\circ} \mathrm{C}$.

\subsection{Cell concentration}

It is possible to label lymphocyte suspensions ranging in concentration from $1 \times 10^{6}-1 \times 10^{8}$ cells $/ \mathrm{ml}$ and any cellular toxicity associated with lower cell concentrations can be overcome by adding molecules containing free amino groups to the labelling buffer (see below).

\subsection{Labelling volume}

Typically lymphocyte labelling is performed with cell suspensions of 1-2 $\mathrm{ml}$, as this volume allows for the rapid mixing of the dye throughout the cell suspension. 


\subsection{Labelling buffer}

Several studies have shown that CFSE labelling can be toxic for cells $[5,6]$, not just in terms of cell death but also in terms of cell function. For example, apparent cell viability can be unaffected by the labelling procedure but the ability of the cells to proliferate can be seriously compromised. This problem can be easily overcome by labelling cells in tissue culture medium containing high concentrations of protein, the medium and protein providing a high concentration of free amino groups in the labelling solution. It is thought that the free amino groups prevent extracellular CFSE from over-labelling cell surface proteins and interfering with cell proliferation [5,7]. We currently use RPMI1640 tissue culture medium containing $10 \%$ foetal calf serum (FCS) as the labelling solution to limit the toxicity of the labelling procedure [7].

\subsection{Washing}

Typically, after labelling lymphocytes with CFSE and CTV, to remove excess dye the cells are washed three times by centrifugation with medium containing high protein content.

\subsection{Spectral spill over of brightly labelled cells}

The fluorescence intensity of dye-labelled cells declines significantly in the first few days following labelling but particularly during the first 24 hours. Although this change does not affect the ability to track cell proliferation, as the fluorescence decrease is proportionally uniform across all division peaks, the high level of fluorescence achievable with CFSE and CTV means there can be significant spectral "spill over" of fluorescence into spectrally adjacent detector channels of flow cytometers, particularly within these first few days. This is particularly notable for CFSE "spill over" into the PE detector and CTV spill over into the Pacific Orange detector of flow cytometers and is, therefore, something that should be taken into consideration when designing experiments that require very brightly labelled cells.

With these technical considerations taken into account and appropriate methods used to label lymphocyte with CFSE and CTV, cell proliferation experiments can easily detect 8 divisions (i.e., 9 distinct fluorescence peaks) at relatively high resolution (Figure 3) and can potentially resolve additional divisions before interference from cell autofluorescence.

\section{Experimental and biological variables that can affect cell division resolution}

Under identical experimental conditions, replicate samples of lymphocytes labelled with CFSE or CTV generate highly reproducible proliferation profiles (Figure 4). However, it should be noted that in vitro cell proliferation assays are acutely sensitive to starting cell concentrations, as cell density can profoundly influence initiation of proliferation. Furthermore, despite using rigorous procedures to label lymphocytes with CFSE and CTV, invariably the resolution of cell division peaks detected by these two dyes tends to decrease as cell division number increases, suggesting that other factors apart from the labelling technique play a role in division resolution. Indeed, over the years we have noted several experimental and biological variables that can impact on cell dye fluorescence and, therefore, represent potential considerations when analysing dye dilution data sets.

\subsection{Lymphocyte subset differences}

When unfractionated populations of lymphocytes from different lymphoid organs are labelled with CFSE, CTV or Cell Proliferation Dye eFluor 670 (CPD) we have noted that lymphocyte subsets within these populations label with different fluorescence intensities [7]. Most notably, B cells tend to label with higher levels of CPD and CTV than T cells (Figure 5a, left panels). This finding highlights the importance of defining lymphocyte subsets with labelled monoclonal antibodies to delineate target populations precisely in order to achieve the least fluorescence variance of dye labelled cells and, therefore, the best division resolution using the fluorescent dyes. We have also observed that in general, B cells tend to have poorer division resolution than $\mathrm{T}$ cells, with the variance of fluorescent division peaks being significantly broader with $\mathrm{B}$ cells compared to $\mathrm{T}$ cells (this is highlighted in Figure 5a, right panels). The reason for this 


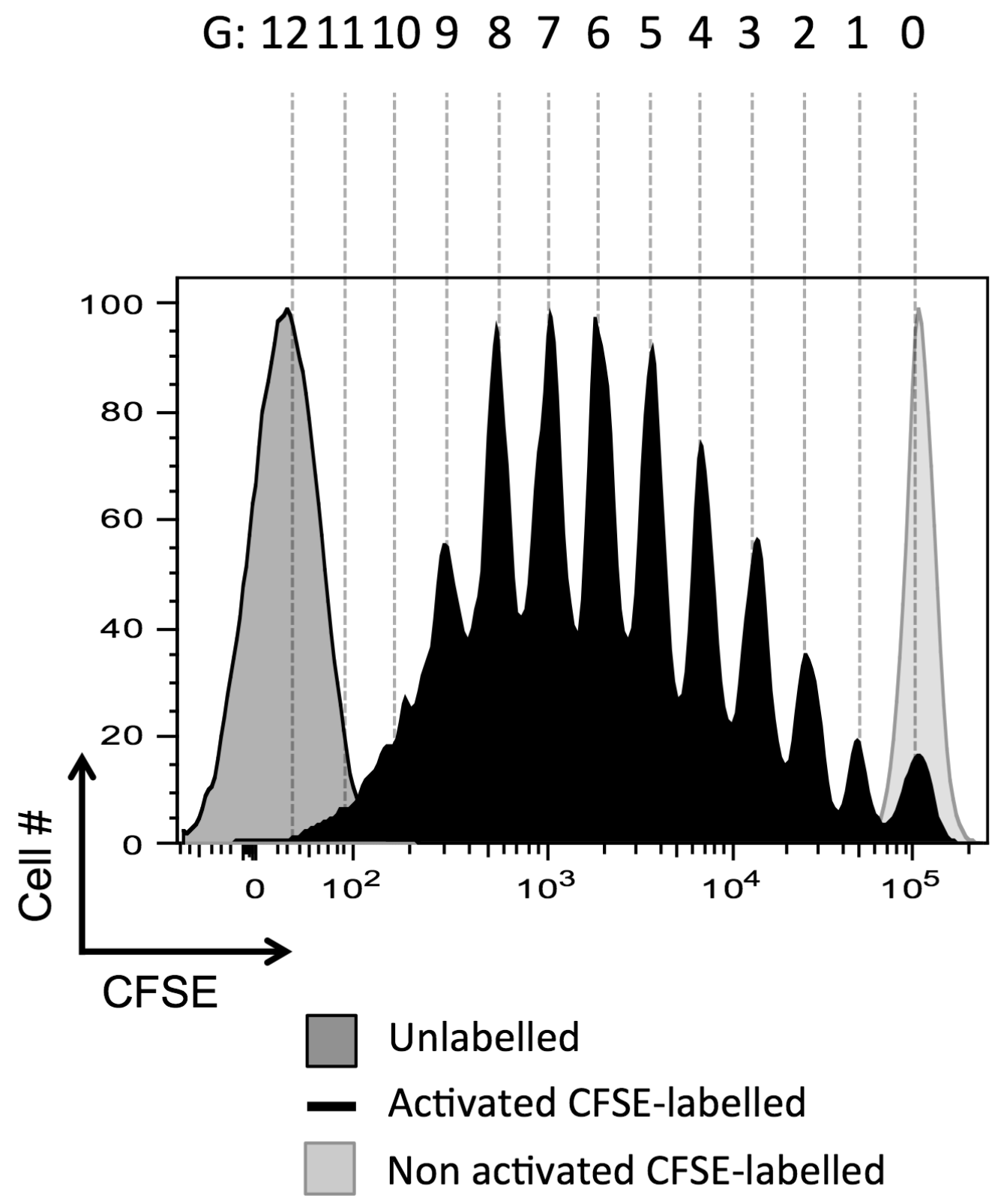

FIGURE 3. High level CFSE-labelling can measure $>8$ lymphocyte divisions. Spleen cells from T cell receptor (TCR) transgenic mice were labelled with $80 \mu \mathrm{M}$ CFSE in RPMI1640 medium supplemented with 10\% FCS and stimulated with specific antigen [7] in an in vivo assay for 4 days and CD8 + T cells analysed by flow cytometry for dye dilution. In addition, unstimulated CFSE-labelled CD8+ T cells and unlabelled CD8+ $\mathrm{T}$ cells were used to monitor undivided cell fluorescence and cell autofluorescence. 
a
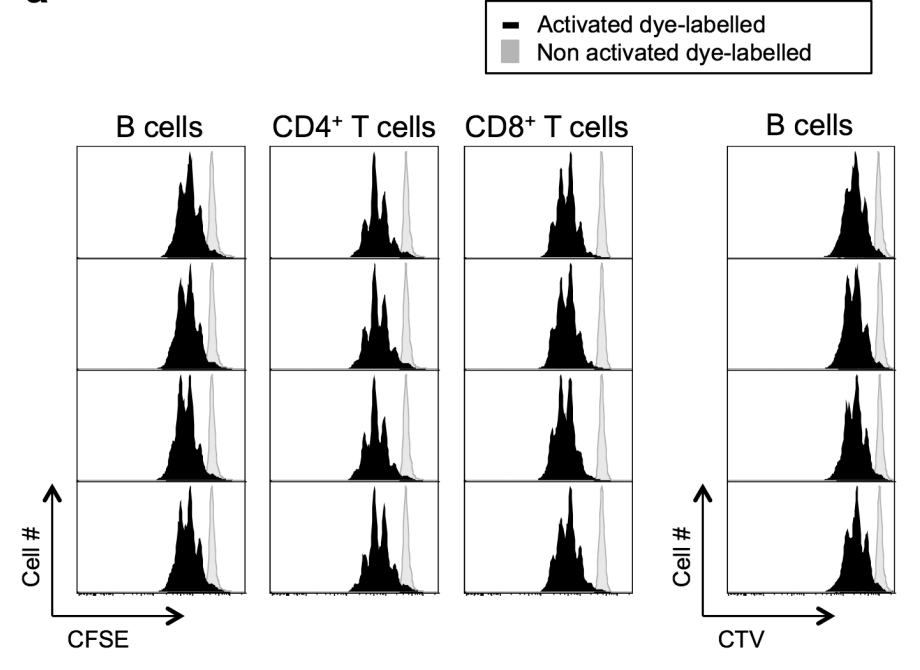

$\mathrm{CD}^{+} \mathrm{T}$ cells $\mathrm{CD}^{+} \mathrm{T}$ cells

b
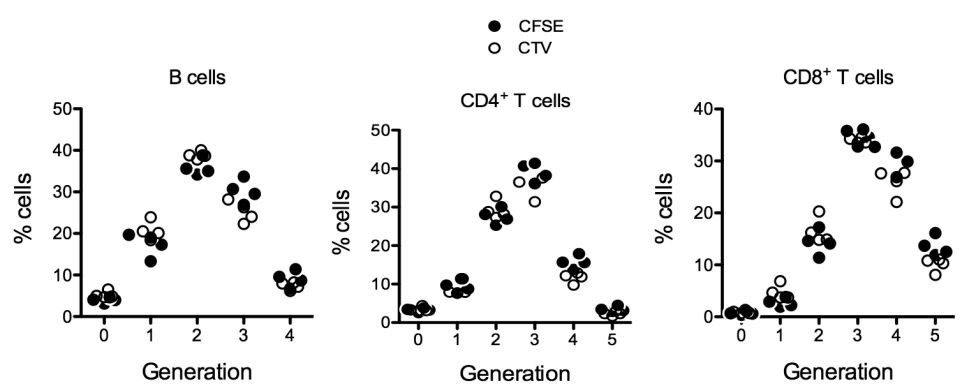

C

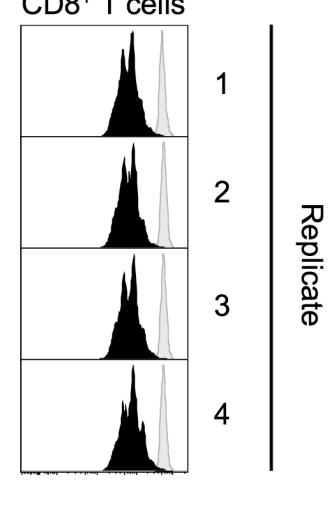

C

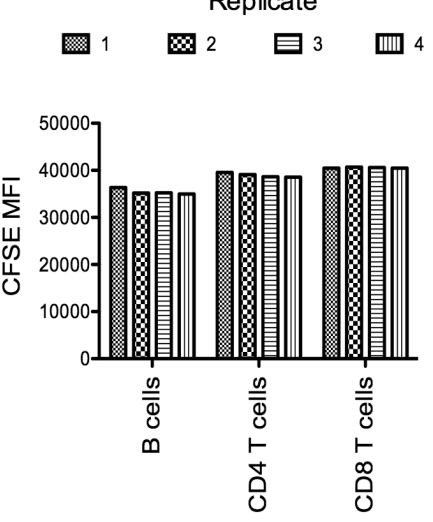

Figure 4. Reproducibility of CFSE and CTV-generated division profiles. Spleen cells from mice were split in to 8 identical aliquots and 4 separate aliquots labelled with $10 \mu \mathrm{M}$ of CFSE and the remaining 4 aliquots labelled with $10 \mu \mathrm{M}$ CTV in RPMI1640 medium supplemented with 10\% FCS. Each of the 8 dye-labelled spleen cell samples were cultured independently with or without multiple mitogens [7] in vitro for 3 days and $\mathrm{B}$ cells, CD4+ $\mathrm{T}$ cells and CD8 $+\mathrm{T}$ cells analysed by flow cytometry for dye dilution. a: Replicate samples of $\mathrm{B}$ cells, CD4+ T cells and CD8+ T cells from spleen cell cultures were assessed for CFSE or CTV fluorescence intensity after 3 days culture. Data shows low variability in division profiles between intra-dye and inter-dye replicates. b: \% of cells in each division peak within each dye-labelled cell subset across all replicates from data depicted in a. Data shows low variability in the proportion of cells detected in each cell division detected between intra-dye and inter-dye replicates. c: Mean fluorescence intensity (MFI) of CFSE or CTV in cell subset replicates in unstimulated spleen cells from data depicted in a showing low variability in fluorescence of replicate samples labelled with each dye. 
appears to be, at least in part, due to the capacity of B cells to take up dye from surrounding dyelabelled cells to a greater degree than $\mathrm{T}$ cells, which results in an increase in division peak variance and consequently decreased division resolution [7] (see dye transfer below).

\subsection{In vitro versus in vivo assays}

Fluorescent dye-labelled lymphocytes can be used to initiate both in vitro and in vivo experiments from the same sample, and the cell fluorescence profiles of dividing cell populations are identical in both experimental formats [34]. Divided cells can be monitored in vivo for extended periods of time and differences in division behaviour, location and survival between lymphocyte subsets monitored [33,37]. However, the resolution of cell division fluorescent peaks is generally better in in vivo assays compared to in vitro assays, particularly when comparing B cell division profiles [7]. We suspect that this difference is a consequence of dye transfer between cells altering division peak fluorescence variance, since dye transfer would be more pronounced in in vitro cultures than in an in vivo setting. Such an effect would result in fluorescence variance and hence division resolution being better in in vivo assays.

\subsection{Cell autofluorescence}

The variance of cell autofluorescence is not uniform across all spectral channels of a flow cytometer. In particular, autofluorescence of cells detected in the channels used for CPD and CTV measurement has broader variance than the autofluorescence of cells in the channel used for CFSE detection [7] (Figure $5 \mathrm{~b})$. We have found that this variance in autofluorescence correlates with the poorer resolution of fluorescent division peaks at later cell divisions, suggesting that as the fluorescence intensity of proliferating dye-labelled cells approaches cell autofluorescence levels they will be influenced more by their autofluorescence variability. An additional consideration here is that when cells are activated they tend to become more autofluorescent than non-activated cells, particularly in the CTV detecting channel, which can compromise the ability of CTV to detect later cell divisions [7] (Figure 5b). These effects of cell autofluorescence on division peak resolution can be reduced by increasing the initial fluorescence intensity of the dye labelled cells by using higher dye concentrations, which allows more divisions to be discretely observed before cell fluorescence approaches autofluorescence levels [7] (Figure 5a, right panels).

\subsection{Dye transfer}

Most fluorescent dyes have some ability to transfer to surrounding cells even after extensive washing of labelled lymphocytes. This appears to be due to both dye-specific and cell intrinsic properties. For example, we have observed that CPD transfers more than CFSE and CFSE transfers more than CTV between dye labelled and unlabelled lymphocytes [7] (Figure 5c). We have also observed that B cells tend to acquire more dye from surrounding dye-labelled cells compared to T cells [7] (Figure 5c). It should be noted that $\mathrm{B}$ cells are known to transfer cell components more readily to bystander cells than $\mathrm{T}$ cells, particularly when they are activated $[38,39]$.

\section{Concluding remarks}

Vital fluorescent dyes, such as CFSE and CTV, provide excellent tools to assess and mathematically model lymphocyte proliferation. In order to obtain the optimum results with dye-dilution proliferation assays and associated mathematical modelling of the data generated, several experimental and biological parameters should be taken into account. Methodologically, lymphocyte populations should be labelled as quickly as possible with high concentrations $(10-80 \mu \mathrm{M})$ of the fluorescent dyes in a labelling medium that yields uniformly brightly labelled cells with minimal dye toxicity issues. With current standard flow cytometers, this will result in an initial fluorescence intensity of undivided cells that is capable of detecting up to 12 cell divisions before the dilution of the dye reaches cell autofluorescence levels. Lymphocyte subsets should be delineated as specifically as possible when analysing dye-dilution data to overcome subset differences in dye uptake. Ideally, in order to accurately gauge the background 
a

Cell differences and increasing

CFSE concentration

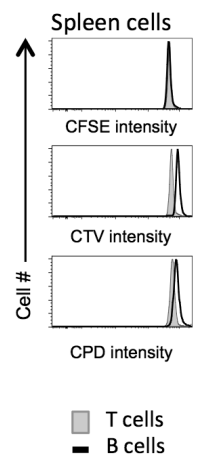

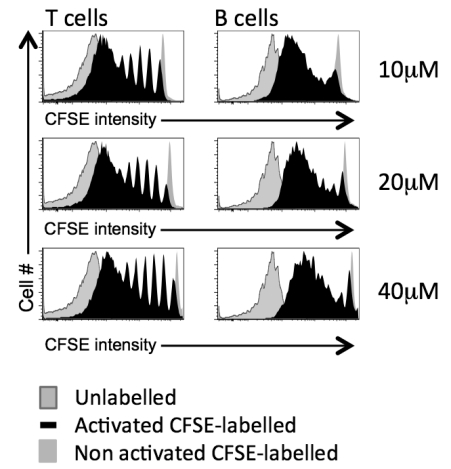

b

Autofluorescence differences

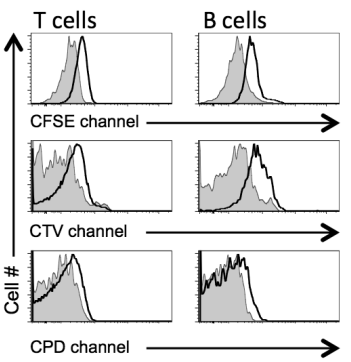

Non activated cells

- Activated cells
C

Dye transfer

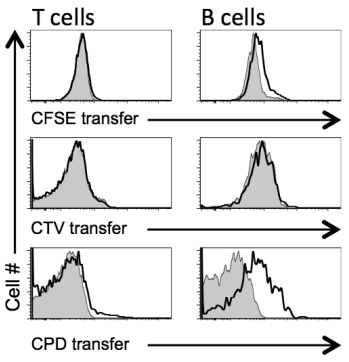

Recipients alone

Recipients with donors

FIGURE 5. Factors contributing to the resolution of fluorescent division peaks with different fluorescent dyes. Spleen cells from mice were labelled with $0-40 \mu \mathrm{M}$ of CFSE, CTV and/or CPD in RPMI1640 medium supplemented with 10\% FCS, cultured with or without multiple mitogens [7] in vitro for 1-4 days and $\mathrm{T}$ cells and $\mathrm{B}$ cells analysed by flow cytometry for fluorescent properties. a, left panels: Unstimulated $\mathrm{T}$ and B cells were simultaneously co-labelled with all three fluorescent dyes and subset differences in dye fluorescence intensity assessed. a, right panels: $\mathrm{T}$ and $\mathrm{B}$ cells labelled with 10, 20 and $40 \mu \mathrm{M}$ CFSE and stimulated in vitro for 4 days were assessed for CFSE fluorescence intensity, the data showing subset differences in division peak resolution and increased resolution of later cell division peaks with higher initial CFSE dye labelling concentrations. b: Autofluorescence of activated and non-activated T and B cells. c: CFSE, CTV and CPD transfer from dye-labelled lymphocytes (donors) to unlabelled T and $\mathrm{B}$ cells (recipients) during in vitro culture.

autofluorescence that dividing cells will reach after multiple cell divisions, activated unlabelled cells should be used as the autofluorescence control, since these cells often exhibit higher levels of autofluorescence than non-activated or resting lymphocytes. While controlling these technical variables will help optimise the detection of lymphocyte cell divisions, they will only have a limited effect on other biological and experimental parameters that can influence cell division resolution, such as the spontaneous transfer of fluorescent dyes to bystander cells, intrinsic lymphocyte subset variability and inherent differences in the cell division data obtained from in vitro versus in vivo experiments.

Acknowledgements. This work was supported by a Project Grant to BQ and CP and a Program Grant to CP from the National Health and Medical Research Council (NHMRC) of Australia. We wish to thank Harpreet Vohra and Michael Devoy for their excellent maintenance of the JCSMR FACS laboratory.

\section{References}

[1] S.A. Weston, C.R. Parish. New fluorescent dyes for lymphocyte migration studies. Analysis by flow cytometry and fluorescence microscopy. J Immunol Methods, 133 (1990), no. 1, 87-97.

[2] A.B. Lyons, C.R. Parish. Determination of lymphocyte division by flow cytometry. J Immunol Methods, 171 (1994) no. $1,131-7$.

[3] A.B. Lyons. Divided we stand: tracking cell proliferation with carboxyfluorescein diacetate succinimidyl ester. Immunol Cell Biol, 77 (1999), no. 6 , 509-15. 
[4] R.E. Nordon, S.S. Ginsberg, C.J. Eaves. High-resolution cell division tracking demonstrates the FLt3-ligand-dependence of human marrow CD34+CD38- cell production in vitro. Br J Haematol, 98 (1997), no. 3, 528-39.

[5] C.R. Parish, M.H. Glidden, B.J. Quah, H.S. Warren. Use of the intracellular fluorescent dye CFSE to monitor lymphocyte migration and proliferation. Curr Protoc Immunol, 84 (2009), 4.9.1-4.9.13.

[6] J. Lastóvicka, V. Budinsky, R. Spisek, J. Bartunkova. Assessment of lymphocyte proliferation: CFSE kills dividing cells and modulates expression of activation markers. Cell Immunol, 256 (2009), no. 1-2, 79-85.

[7] B.J. Quah, C.R. Parish. New and improved methods for measuring lymphocyte proliferation in vitro and in vivo using CFSE-like fluorescent dyes. J Immunol Methods, 379 (2012), no. 1-2, 1-14.

[8] B.J. Quah, H.S. Warren, C.R. Parish. Monitoring lymphocyte proliferation in vitro and in vivo with the intracellular fluorescent dye carboxyfluorescein diacetate succinimidyl ester. Nat Protoc, 2 (2007), no. 9, 2049-56.

[9] B.J. Quah, C.R. Parish. The use of carboxyfluorescein diacetate succinimidyl ester (CFSE) to monitor lymphocyte proliferation. Journal of visualized experiments : JoVE, 44 (2010).

[10] R.N. Morrison, A.B. Lyons, B.F. Nowak, J.D. Hayball. Snapper (Pagrus auratus) leucocyte proliferation is synergistically enhanced by simultaneous stimulation with LPS and PHA. Fish Shellfish Immunol, 16 (2004), no. 3, 307-19.

[11] T. Holyoake, X. Jiang, C. Eaves, A. Eaves. Isolation of a highly quiescent subpopulation of primitive leukemic cells in chronic myeloid leukemia. Blood, 94 (1999), no. 6, 2056-64.

[12] J.E. Ueckert, G. Nebe von-Caron, A.P. Bos, P.F. ter Steeg. Flow cytometric analysis of Lactobacillus plantarum to monitor lag times, cell division and injury. Lett Appl Microbiol, 25 (1997), no. 4, 295-9.

[13] P.D. Hodgkin, J.H. Lee, A.B. Lyons. B cell differentiation and isotype switching is related to division cycle number. J Exp Med, 184 (1996), no. 1, 277-81.

[14] A.V. Gett, P.D. Hodgkin. Cell division regulates the $T$ cell cytokine repertoire, revealing a mechanism underlying immune class regulation. Proc Natl Acad Sci U S A, 95 (1998), no. 16, 9488-93.

[15] J. Hasbold, A.B. Lyons, M.R. Kehry, P.D. Hodgkin. Cell division number regulates IgG1 and IgE switching of B cells following stimulation by CD40 ligand and IL-4. Eur J Immunol, 28 (1998), no. 3, 1040-51.

[16] J. Hasbold, P.D. Hodgkin. Flow cytometric cell division tracking using nuclei. Cytometry, 40 (2000), no. 3, $230-7$.

[17] S. Blake, T.P. Hughes, G. Mayrhofer, A.B. Lyons. The Src/ABL kinase inhibitor dasatinib (BMS-354825) inhibits function of normal human T-lymphocytes in vitro. Clin Immunol, 127 (2008), no. 3, 330-9.

[18] S.J. Blake, A.B. Lyons, T.P. Hughes. Nilotinib inhibits the Src-family kinase LCK and T-cell function in vitro. J Cell Mol Med, 13 (2009), no. 3, 599-601.

[19] C.K. Fraser, S.J. Blake, K.R. Diener, A.B. Lyons, M.P. Brown, T.P. Hughes, J.D. Hayball. Dasatinib inhibits recombinant viral antigen-specific murine CD4+ and CD8+T-cell responses and NK-cell cytolytic activity in vitro and in vivo. Exp Hematol, 37 (2009), no. 2, 256-65.

[20] R.E. Nordon, M. Nakamura, C. Ramirez, R. Odell. Analysis of growth kinetics by division tracking. Immunol Cell Biol, 77 (1999), no. 6, 523-9.

[21] R.J. De Boer, V.V. Ganusov, D. Milutinovic, P.D. Hodgkin, A.S. Perelson. Estimating lymphocyte division and death rates from CFSE data. Bull Math Biol, 68 (2006), no. 5, 1011-31.

[22] E.D. Hawkins, M. Hommel, M.L. Turner, F.L. Battye, J.F. Markham, P.D. Hodgkin. Measuring lymphocyte proliferation, survival and differentiation using CFSE time-series data. Nat Protoc, 2 (2007), no. 9, $2057-67$.

[23] R.E. Nordon, K.H. Ko, R. Odell, T. Schroeder. Multi-type branching models to describe cell differentiation programs. J Theor Biol, 277 (2011), no. 1, 7-18.

[24] H.Y. Lee, E. Hawkins, M.S. Zand, T. Mosmann, H. Wu, P.D. Hodgkin, A.S. Perelson. Interpreting CFSE obtained division histories of $B$ cells in vitro with Smith-Martin and cyton type models. Bull Math Biol, 71 (2009), no. 7, 1649-70.

[25] S.J. Blake, T.P. Hughes, A.B. Lyons. Drug-interaction studies evaluating T-cell proliferation reveal distinct activity of dasatinib and imatinib in combination with cyclosporine A. Exp Hematol, (2012).

[26] H.T. Banks, K.L. Sutton, W.C. Thompson, G. Bocharov, M. Doumic, T. Schenkel, J. Argilaguet, S. Giest, C. Peligero, A. Meyerhans. A new model for the estimation of cell proliferation dynamics using CFSE data. J Immunol Methods, 373 (2011), no. 1-2, 143-60.

[27] H.T. Banks, K.L. Sutton, W.C. Thompson, G. Bocharov, D. Roose, T. Schenkel, A. Meyerhans. Estimation of cell proliferation dynamics using CFSE data. Bull Math Biol, 73 (2011), no. 1, 116-50.

[28] C.R. Parish. Fluorescent dyes for lymphocyte migration and proliferation studies. Immunol Cell Biol, 77 (1999), no. 6, 499-508.

[29] R. Zeng, R. Spolski, E. Casas, W. Zhu, D.E. Levy, W.J. Leonard. The molecular basis of IL-21-mediated proliferation. Blood, 109 (2007), no. 10, 4135-42.

[30] P.K. Wallace, J.D. Tario, Jr., J.L. Fisher, S.S. Wallace, M.S. Ernstoff, K.A. Muirhead. Tracking antigen-driven responses by flow cytometry: monitoring proliferation by dye dilution. Cytometry, Part A, 73 (2008), no. 11, 1019-34.

[31] Y. Singh, J. Dyson, O.A. Garden. Use of SNARF-1 to measure murine T cell proliferation in vitro and its application in a novel regulatory $T$ cell suppression assay. Immunol Letters, 140 (2011), no. 1-2, 21-9.

[32] D.A. Fulcher, A.B. Lyons, S.L. Korn, M.C. Cook, C. Koleda, C. Parish, B. Fazekas de St Groth, A. Basten. The fate of self-reactive $B$ cells depends primarily on the degree of antigen receptor engagement and availability of $T$ cell help. J Exp Med, 183 (1996), no. 5, 2313-28.

[33] A.B. Lyons. Pertussis toxin pretreatment alters the in vivo cell division behaviour and survival of B lymphocytes after intravenous transfer. Immunol Cell Biol, 75 (1997), no. 1, 7-12. 
[34] A.B. Lyons. Analysing cell division in vivo and in vitro using flow cytometric measurement of CFSE dye dilution. J Immunol Methods, 243 (2000), no. 1-2, 147-54.

[35] A.B. Lyons., J. Hasbold, P.D. Hodgkin. Flow cytometric analysis of cell division history using dilution of carboxyfluorescein diacetate succinimidyl ester, a stably integrated fluorescent probe. Method Cell Biol, 63 (2001), 375-98.

[36] A.B. Lyons, K.V. Doherty. Flow cytometric analysis of cell division by dye dilution. Current protocols in cytometry, (2004), Chapter 9: Unit 911.

[37] A.B. Lyons, M. Watkins, C.C. Simpson, H. Konrad Muller. Modulation of lymphocyte migration to the murine spleen after marginal zone macrophage phagocytosis of blood-borne particulate material. Immunol Invest, 35 (2006), no. 1, 75-92.

[38] B.J. Quah, V.P. Barlow, V. McPhun, K.I. Matthaei, M.D. Hulett, C.R. Parish. Bystander B cells rapidly acquire antigen receptors from activated B cells by membrane transfer. Proc Natl Acad Sci U S A, 105 (2008), no. 11, 4259-64.

[39] G. Chaudhri, B.J. Quah, Y. Wang, A.H. Tan, J. Zhou, G. Karupiah, C.R. Parish. T cell receptor sharing by cytotoxic T lymphocytes facilitates efficient virus control. Proc Natl Acad Sci U S A, 106 (2009), no. 35, 14984-14989. 